\title{
Biopsia endobiliar con pinza por acceso percutáneo: primera experiencia en Uruguay
}

Joaquín García*, Alonzo Rodríguez, Nelson di Trápanił, Marta Otero§

\section{Resumen}

Presentamos la primera experiencia en Uruguay de una biopsia endobiliar con pinza por acceso percutáneo que realizáramos en una paciente con estenosis biliar hiliar de probable causa neoplásica. Hasta nuestro conocimiento, no existen reportes de esta técnica en Uruguay.

La difusión de la disponibilidad de esta técnica en nuestro medio es de gran importancia, ya que permite al equipo médico tratante disponer de una nueva herramienta para el manejo diagnóstico de las estenosis biliares.

Palabras clave: Biopsia

Estenosis

Técnica de diagnóstico

Enfermedades del conducto biliar

\section{Historia clínica}

Paciente de sexo femenino, 61 años, tabaquista, dislipémica.

Consultó en puerta de emergencia del Hospital de Clínicas por cuadro de ictericia, coluria y acolia de una semana de evolución, con intenso prurito. Negó fiebre y repercusión general.

$\mathrm{Al}$ examen físico presentó ictericia universal y un abdomen globuloso, sin dolor a la palpación, con signo de Bard y Pick positivo.

La paraclínica al ingreso evidenció elementos de colestasis (bilirrubina total de $10,12 \mathrm{mg} / \mathrm{dL}$, a expensas de la bilirrubina directa de $8,98 \mathrm{mg} / \mathrm{dL}$; fosfatasa alcalina 444 UI/L; GGT 498 UI/L) y signos de lesión hepatocítica (TGO $171 \mathrm{UI} / \mathrm{L}$ y TGP $252 \mathrm{UI} / \mathrm{L}$ ).

Marcadores tumorales: CA 19-9: 980,5 UI/ml.

Se solicitó ecografía y tomografía computada de abdomen con contraste intravenoso en las que se confirmó la dilatación de la vía biliar intrahepática, con estenosis en el conducto hepático principal, inmediatamente distal al confluente biliar superior. La vesícula estaba distendida y presentaba paredes engrosadas, sin un claro plano de separación con el hígado.

Con el planteo de una estenosis alta de la vía biliar a nivel hiliar, de causa neoplásica maligna, secundaria a un tumor de vesícula biliar, se ingresó a la paciente a sala para completar la valoración y el tratamiento.

Se intentó realizar un drenaje biliar endoscópico para disminuir la colestasis, sin éxito, por lo que se decidió realizar un drenaje biliar percutáneo.

Luego de recabar el consentimiento informado de la paciente, se colocó un drenaje biliar percutáneo externo-interno por acceso derecho, según técnica descrita por Patel $^{(1)}$, previa antibioticoterapia con ampicilina-sulbac-

\footnotetext{
* Asistente del Departamento Clínico de Imagenología. † Prof. Adjunto del Departamento Clínico de Imagenología. $\ddagger$ Ex Profesor del Departamento Clínico de Imagenología. $\S$ Prof. Agregada del Departamento de Anatomía Patológica. Hospital de Clínicas "Dr. Manuela Quintela". Montevideo, Uruguay. Los autores declaran no tener conflictos de interés.

Este trabajo cuenta con el consentimiento informado del paciente para su publicación Recibido: $11 / 6 / 19$ Aprobado: 9/9/19
} 


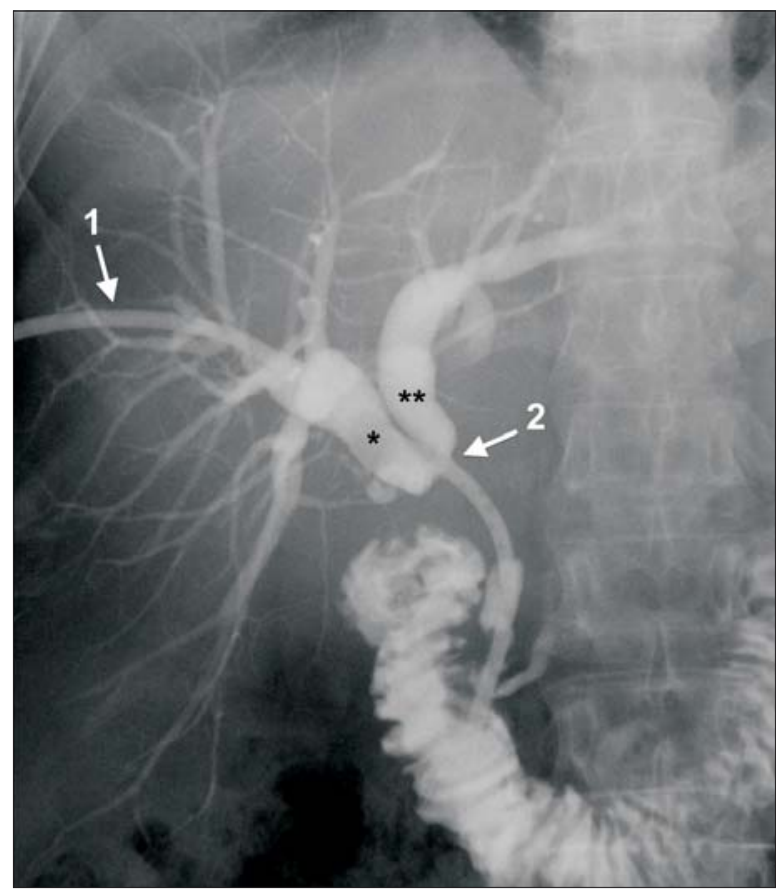

Figura 1. Drenaje biliar percutáneo externo-interno: acceso derecho. Catéter de drenaje biliar externo-interno que ingresa al árbol biliar derecho, con trayecto transestenótico y extremo distal en la luz duodenal. La colangiografía anterógrada demuestra la dilatación de la vía biliar intrahepática con una estenosis abrupta a nivel del conducto hepático común, sin compromiso de los conductos hepáticos derecho e izquierdo.

1: Catéter de drenaje biliar. 2: Sitio de estenosis.

*: Conducto hepático derecho. **: Conducto hepático izquierdo.

tam y bajo sedoanalgesia y anestesia local, siguiendo las pautas de la Sociedad de Radiología Intervencionista ${ }^{(2)}$. La colangiografía anterógrada transcatéter demostró la estenosis abrupta del conducto hepático común a menos de $2 \mathrm{~cm}$ del confluente biliar superior (Bismuth II) (figura 1).

Frente a la falta de confirmación histológica de la causa de estenosis, se decidió, en conjunto con el equipo tratante, realizar una biopsia endobiliar percutánea como primera opción para evitar una cirugía diagnóstica.

Luego de recabar nuevamente el consentimiento de la paciente, se intercambió bajo guías el drenaje biliar interno-externo derecho por una vaina $9 \mathrm{Fr}$, a través de la cual se introdujo una pinza de biopsia de 2,3 $\mathrm{mm}$ de diámetro (Gaototec, ATE Med, China) (figura 2).

Bajo control radioscópico se enfrentó la pinza al sitio de obstrucción y se tomaron muestras del sector central de la estenosis en cinco oportunidades mediante la técnica de cross and push ${ }^{(1)}$ (figura 3), obteniendo frag- mentos sólidos, blanquecinos, de entre 1-3 $\mathrm{mm}$ de diámetro.

Durante el procedimiento se realizó un nuevo drenaje biliar percutáneo externo-interno por acceso izquierdo para manejo futuro de la estenosis biliar con prótesis metálicas internas.

El procedimiento fue bien tolerado y no existieron complicaciones.

La paciente tuvo una buena evolución en sala, con rápida mejoría del síndrome pigmentario, sin dolor ni fiebre, tolerando vía oral desde el momento del procedimiento, sin hemobilia ni melenas.

Se otorgó alta a domicilio, a la espera de recambio de los drenajes biliares externos-internos por prótesis metálicas internas.

\section{Anatomía patológica}

Las muestras fueron procesadas con el procedimiento de rutina: fijación en formol tamponado a $10 \%$, inclusión en parafina y cortes de 4 micras de espesor coloreados con hematoxilina-eosina.

El estudio definitivo demostró una biopsia de la pared de vía biliar infiltrada por un adenocarcinoma pobremente diferenciado. Los hallazgos morfológicos en el contexto clínico de la paciente, sumado al engrosamiento parietal vesicular, apoyan el diagnóstico clínico de tumor de vesícula biliar (figura 4).

\section{Discusión}

El estudio y manejo de las estenosis biliares ha tenido importantes avances en los últimos años, principalmente de la mano del desarrollo de las técnicas de imagen no invasivas que permiten definir la localización y extensión de la estenosis (US, CT, RM). Sin embargo, el diagnóstico diferencial entre estenosis biliares benignas y malignas es en algunos casos desafiante, ya que en ambos tipos pueden presentar similar semiología imagenológica. Además, los tumores originados del epitelio de la vía biliar son, en un alto porcentaje, demasiado pequeños para visualizarlos por técnicas de imagen ${ }^{(3)}$.

La confirmación histológica de una estenosis de sospecha neoplásica por técnicas miniinvasivas permite iniciar un tratamiento oncoespecífico sin compromisos médico legales, además de evitar un abordaje quirúrgico con objetivos diagnósticos o un tratamiento quirúrgico radical en casos en que no estuviera indicado (por ejemplo, linfoma, infiltración biliar por adenomegalias metastásicas).

La biopsia endobiliar transparietohepática con pinza de biopsia fue descrita por primera vez en $1980^{(4)}$. En las últimas dos décadas se produjo un marcado aumento de las publicaciones de series de casos, probablemente asociado a los avances descritos en la técnica y la mejora de 


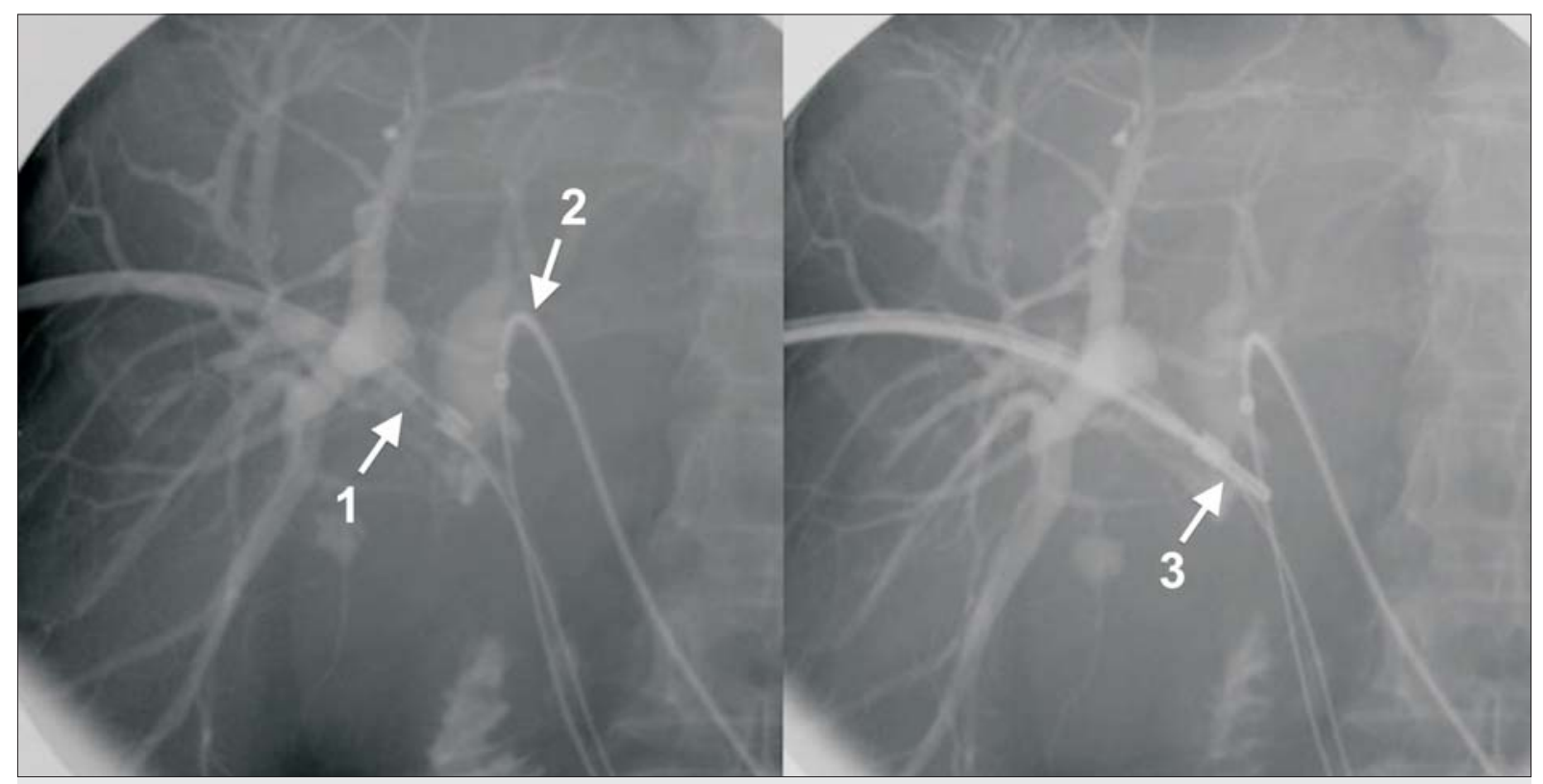

Figura 2. Introducción de la pinza de biopsia. Se recambió el drenaje catéter de drenaje biliar interno-externo por una vaina con punta radioopaca (1) y a través de esta se introduce la pinza de biopsia (3), enfrentándola al sector central de la estenosis. 2: Introductor de acceso biliar izquierdo.

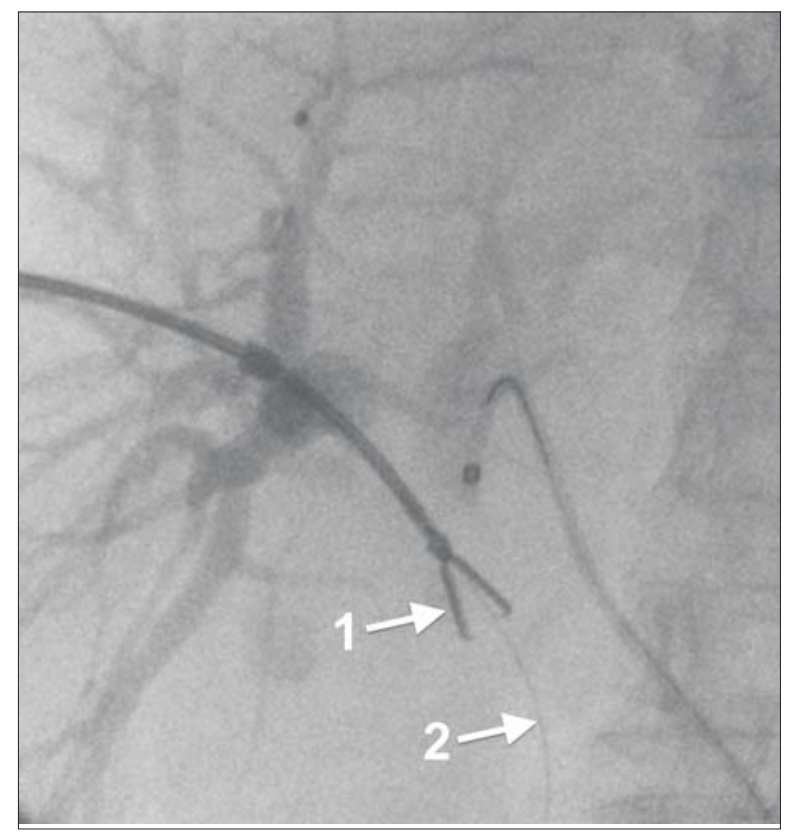

Figura 3. Biopsia endobiliar: técnica cross and push. La pinza de biopsia (1) se avanza abierta a través del sector central de la estenosis, para luego cerrarla y obtener la muestra. La guía de seguridad (2) mantiene el trayecto transestenótico y marca el sector central de la estenosis. los materiales utilizados en radiología intervencionis$\mathrm{ta}^{(1)}$.

El método tiene un buen rendimiento diagnóstico, con grandes series que demuestran alta sensibilidad y especificidad, con un excelente valor predictivo positivo. Sin embargo, el valor predictivo negativo es bajo (tabla 1).

En un estudio dirigido a valorar los factores asociados al rendimiento de la biopsia endobiliar con pinza de biopsia por acceso percutáneo, Park y colaboradores ${ }^{(3)}$ demostraron que la sensibilidad y especificidad de la técnica son significativamente mayores en obstrucciones hiliares y en tumores originados del epitelio de la vía biliar. Esto podría estar relacionado a la dificultad de enfrentar la pinza de biopsia al sector central de la estenosis en tumores periampulares o del colédoco distal. Además, teniendo en cuenta que la biopsia está dirigida a la superficie del conducto biliar, es lógico pensar que el rendimiento diagnóstico será mayor en tumores originados en el propio epitelio biliar que en estenosis por infiltración/compresión extrínseca.

El método es seguro y agrega muy poco riesgo cuando se asocia a un drenaje biliar percutáneo ${ }^{(1,3,5-9)}$. En su mayoría, las complicaciones son transitorias (hemobilia) o se resuelven en forma miniinvasiva (biloma, pseudoaneurisma de la arteria hepática), y muchas de ellas están relacionadas a la punción hepática para el acceso biliar y no a la propia biopsia endobiliar (tabla 2). No te- 


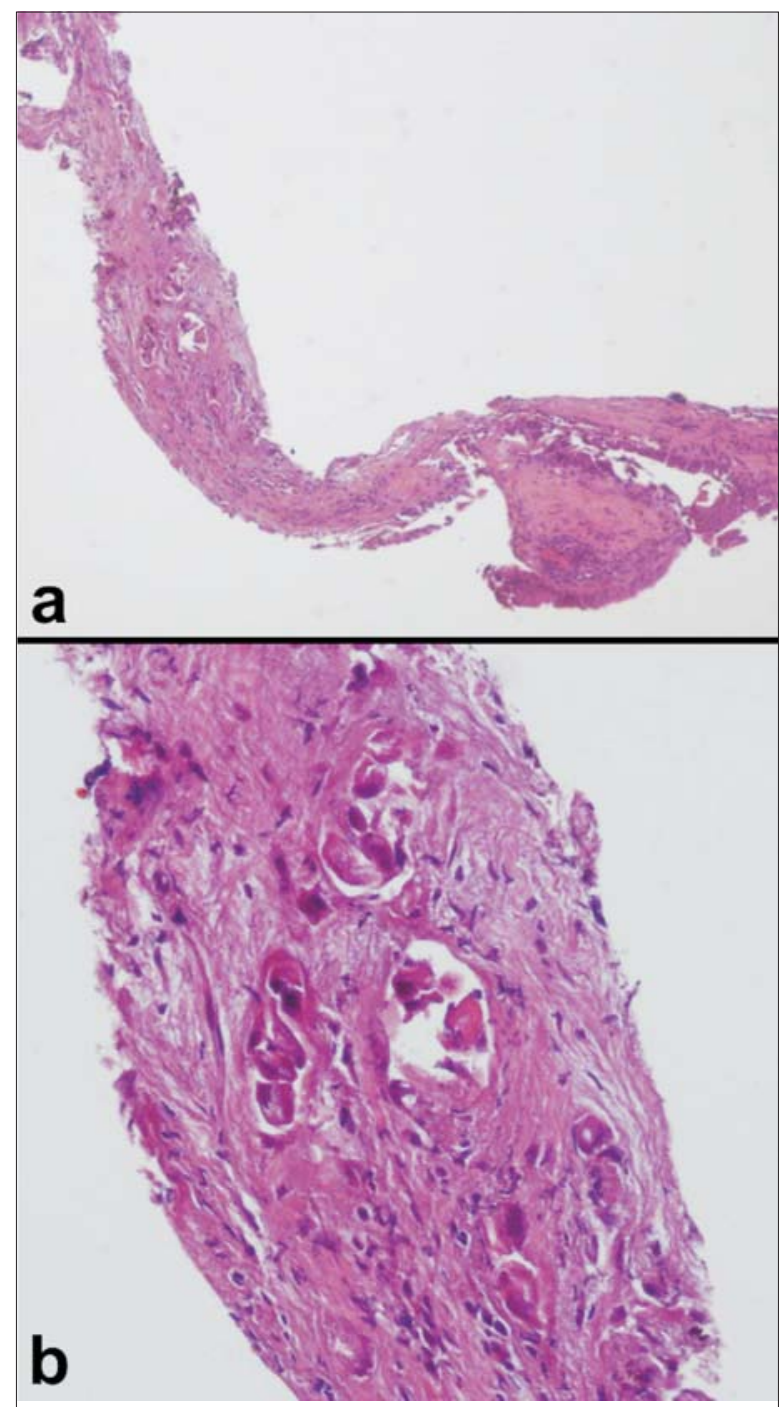

Figura 4. Carcinoma invadiendo la pared de la vía biliar. Microfotografía de la pieza de biopsia.

a) Hematoxilina y eosina. 4x. Carcinoma invadiendo la pared de la vía biliar. Arriba, a la izquierda: nidos tumorales. Abajo, a la derecha: mucosa con epitelio nativo.

b) Hematoxilina y eosina. 10x. Células atípicas en nidos y dispersas con estroma desmoplásico, algunas de ellas vacuoladas.

nemos conocimiento de reportes de muertes vinculadas directamente al procedimiento.

No quedan dudas del rol central de la biopsia dirigida por ecoendoscopía en el manejo de las estenosis biliares, siendo actualmente el método de elección para el diagnóstico de las estenosis periampulares. Tiene como limitantes el alto coste y la necesidad de personal altamente especializado. Su rendimiento diagnóstico en estenosis hiliares o intrahepáticas disminu- ye, y, cuando menos, es comparable al de la biopsia endobiliar con pinza de biopsia por acceso percutáneo ${ }^{(5)}$.

La biopsia bajo visión colangioscópica permite la visualización de la estenosis y la posibilidad de dirigir la biopsia a los sectores de máxima sospecha. Puede realizarse por acceso percutáneo o endoscópico. El primero tiene excelente rendimiento diagnóstico en el colangiocarcinoma, pero requiere de un trayecto transparietohepático de gran calibre para permitir el paso del colangioscopio $^{(10)}$, lo que podría vincularse a mayores complicaciones. Por otra parte, el pequeño tamaño de las muestras obtenidas a través del acceso endoscópico podría ser la causa de las sensibilidades intermedias reportadas en la literatura $(49 \%-77 \%)^{(11,12)}$.

Otras técnicas diagnósticas, como el estudio citológico de la bilis o el cepillado por acceso percutáneo o endoscópico, son poco utilizadas por su bajo rendimiento diagnóstico ${ }^{(13-15)}$.

Creemos que la biopsia endobiliar con pinza por acceso percutáneo tiene un lugar reservado en el algoritmo diagnóstico de las estenosis biliares de probable etiología neoplásica en pacientes que no son candidatos a un tratamiento quirúrgico curativo, especialmente frente a estenosis altas o sospecha clínica de neoplasia de la vía biliar.

\section{Abstract}

The study presents the first experience in Uruguay of a forceps biopsy of biliary ducts via percutaneous catheterization performed in a patient with hilar biliary strictures probably caused by a tumor. As far as we know, no reports on this technique have been published in our country.

It is important to spread the availability of this technique in our country since it provides the medical team with a new tool for the diagnostic handling of biliary strictures.

\section{Resumo}

Apresentamos a primeira experiencia realizada no país de una biopsia endobiliar com pinça por acesso percutâneo, realizada em uma paciente com uma estenose biliar hilar com provável causa neoplásica. Até onde sabemos não existem registros de realização desta técnica no Uruguai.

A difusão da disponibilidade desta técnica no nosso meio tem grande importância pois permite as equipes médicas dispor de uma nova ferramenta para o manejo diagnóstico das estenoses biliares. 


\begin{tabular}{|c|c|c|c|c|c|c|}
\hline Autores & $N$ & $S(\%)$ & $E(\%)$ & VPP (\%) & VPN (\%) & $P D$ \\
\hline Park, et al ${ }^{(3)}$ & 271 & 77,2 & 100 & 100 & 26 & 78,9 \\
\hline Mohkam, et al(5) & 75 & 75 & 100 & 100 & 58 & 78 \\
\hline Fohlen, et a $\left.\right|^{(9)}$ & 50 & 70 & 100 & 100 & 22 & 72 \\
\hline$L i$, et al $\left.\right|^{(6)}$ & 826 & 86,6 & 100 & 100 & 46,5 & $N R$ \\
\hline lerardi, et $\mathrm{al}^{(7)}$ & 40 & 85 & 100 & $N R$ & - & 88,7 \\
\hline Patel, et al ${ }^{(1)}$ & 52 & 93,3 & 100 & 100 & 70 & 94,2 \\
\hline Jung, et al ${ }^{(8)}$ & 130 & 78,4 & 100 & 100 & 15,6 & 79,2 \\
\hline
\end{tabular}

N: número de casos; S: sensibilidad; E: especificidad; VPP: valor predictivo positivo; VPN: valor predictivo negativo; PD: precisión diagnóstica; NR: no reportado.

Tabla 2. Complicaciones reportadas de la biopsia endobiliar con pinza de biopsia por acceso percutáneo.

\begin{tabular}{lcccc}
\hline & & & Complicaciones \\
\cline { 3 - 4 } & $N$ & Total (\%) & Severas & Fatales \\
\hline Park, et al ${ }^{(3)}$ & 271 & $11(4)$ & 1 & 0 \\
Fohlen, et al ${ }^{(9)}$ & 50 & $4(8)$ & 1 & 0 \\
Li, et al $^{(6)}$ & 826 & $39(5)$ & 0 & 0 \\
lerardi, et al $^{(7)}$ & 40 & $15(38)$ & 1 & 0 \\
Patel, et al ${ }^{(1)}$ & 52 & $1(2)$ & 0 & 0 \\
Jung, et al $\left.\right|^{(8)}$ & 130 & $8(6)$ & & 0 \\
\hline
\end{tabular}

\section{Bibliografía}

1. Patel P, Rangarajan B, Mangat K. Improved accuracy of percutaneous biopsy using "Cross and Push" technique for patients suspected with malignant biliary strictures. Cardiovasc Intervent Radiol 2015; 38(4):1005-10.

2. Saad WE, Wallace MJ, Wojak JC, Kundu S, Cardella JF. Quality improvement guidelines for percutaneous transhepatic cholangiography, biliary drainage, and percutaneous cholecystostomy. J Vasc Interv Radiol 2010; 21(6):789-95.

3. Park JG, Jung GS, Yun JH, Yun BC, Lee SU, Han BH, et al. Percutaneous transluminal forceps biopsy in patients suspected of having malignant biliary obstruction: factors influencing the outcomes of 271 patients. Eur Radiol 2017; 27(10):4291-7.

4. Elyaderani MK, Gabriele OF. Brush and forceps biopsy of biliary ducts via percutaneous transhepatic catheterization. Radiology 1980; 135(3):777-8.
Mohkam K, Malik Y, Derosas C, Isaac J, Marudanayagam R, Mehrzad H, et al. Percutaneous transhepatic cholangiographic endobiliary forceps biopsy versus endoscopic ultrasound fine needle aspiration for proximal biliary strictures: a single-centre experience. HPB(Oxford) 2017; 19(6):530-7.

6. Li Z, Li TF, Ren JZ, Li WC, Ren JL, Shui SF, et al. Value of percutaneous transhepatic cholangiobiopsy for pathologic diagnosis of obstructive jaundice: analysis of 826 cases. Acta Radiol 2017; 58(1):3-9.

7. Ierardi AM, Mangini M, Fontana F, Floridi C, De Marchi G, Petrillo M, et al. Usefulness and safety of biliary percutaneous transluminal forceps biopsy (PTFB): our experience. Minim Invasive Ther Allied Technol 2014; 23(2):96-101.

8. Jung GS, Huh JD, Lee SU, Han BH, Chang HK, Cho YD. Bile duct: analysis of percutaneous transluminal forceps biopsy in 130 patients suspected of having malignant biliary obstruction. Radiology 2002; 224(3):725-30. 
9. Fohlen A, Bazille C, Menahem B, Jegonday MA, Dupont B, Le Pennec V, et al. Transhepatic forceps biopsy combined with biliary drainage in obstructive jaundice: safety and accuracy. Eur Radiol 2019; 29(5):2426-35.

10. Nimura Y. Staging of biliary carcinoma: cholangiography and cholangioscopy. Endoscopy 1993; 25(1):76-80.

11. Hartman DJ, Slivka A, Giusto DA, Krasinskas AM. Tissue yield and diagnostic efficacy of fluoroscopic and cholangioscopic techniques to assess indeterminate biliary strictures. Clin Gastroenterol Hepatol 2012; 10(9):1042-6.

12. Siddiqui AA, Mehendiratta V, Jackson W, Loren DE, Kowalski TE, Eloubeidi MA. Identification of cholangiocarcinoma by using the Spyglass Spyscope system for peroral cholangioscopy and biopsy collection. Clin Gastroenterol Hepatol 2012; 10(5):466-71.
13. Savader SJ, Lynch FC, Radvany MG, Kudryk BT, Andrews RT, Geschwind JF, et al. Single-specimen bile cytology: a prospective study of 80 patients with obstructive jaundice. J Vasc Interv Radiol 1998; 9(5):817-21.

14. Tapping CR, Byass OR, Cast JE. Cytological sampling versus forceps biopsy during percutaneous transhepatic biliary drainage and analysis of factors predicting success. Cardiovasc Intervent Radiol 2012; 35(4):883-9.

15. Ponchon T, Gagnon P, Berger F, Labadie M, Liaras A, Chavaillon A, et al. Value of endobiliary brush cytology and biopsies for the diagnosis of malignant bile duct stenosis: results of a prospective study. Gastrointest Endosc 1995; 42(6):565-72.

\section{Contribución de autores}

Joaquín García, https://orcid.org/0000-0003-3404-8558. Concepción, diseño, ejecución, redacción y revisión crítica.

Alonzo Rodríguez, https://orcid.org/0000-0001-6669-9844. Concepción, diseño, ejecución, redacción y revisión crítica.

Nelson di Trápani, https://orcid.org/0000-0003-4599-286X. Ejecución y revisión crítica.

Marta Otero, https://orcid.org/0000-0003-3691-9871. Ejecución y revisión crítica. 